\title{
Spin-isospin Responses of Deformed Neutron-rich Nuclei
}

\section{Kenichi Yoshida*}

Graduate School of Science and Technology, Niigata University, Niigata 950-0913, Japan

E-mail: kvoshidaephys.sc.niigata-u.ac.jp

Deformation effects on the spin-isospin responses of nuclei, in particular, the Gamow-Teller (GT) modes of excitation are investigated in a microscopic framework based on nuclear densityfunctional theory. To describe the low-lying GT states and the GT giant resonance (GTGR) in deformed neutron-rich nuclei, I employ the Skyrme energy-density functionals in the HartreeFock-Bogoliubov calculation for the ground states and in the Quasiparticle Random-Phase Approximation for the excitations. It is found that the fragmentation of the strength distribution in the low excitation-energy region has a strong impact on the $\beta$-decay properties, while the $K$-splitting is small for the GTGR.

The 26th International Nuclear Physics Conference

11-16 September, 2016

Adelaide, Australia

${ }^{*}$ Speaker. 


\section{Introduction}

The study of unstable nuclei has been a major subject in nuclear physics for a couple of decades thanks to the development of the rare-isotope beam technology. Collective mode of excitation emerging in the response of the nucleus to an external field is a manifestation of the interaction and correlation among nucleons. Thus, the isovevtor channel of the interaction or the energy-density functional (EDF), which is crucial for understanding and predicting the properties of unstable nuclei and asymmetric nuclear matter, has been much studied through especially the Gamow-Teller (GT) excitation besides the isovector dipole excitation [ [1, 口, 四, 团].

The GT strength distribution has been extensively investigated experimentally and theoretically not only because of interests in nuclear structure but also because $\beta$-decay half-lives set a time scale of the rapid-neutron-capture process ( $r$-process), and hence determine the production of heavy elements in the universe [5]. The $r$-process path is far away from the stability line, and involves neutron-rich nuclei. They are weakly bound and many of them are expected to be deformed according to the systematic Skyrme-EDF calculations [ [, , $]$ ].

Though the experimental study of the GT strength distribution in neutron-rich nuclei is yet difficult, the progress has been made for the systematic measurement of the $\beta$-decay properties. Recently, $\beta$-decay half-lives of neutron-rich nuclei with $A \simeq 110$ located on the boundary of the $r$-process path were newly measured at RIKEN RIBF [ $[\mathbb{0}, \mathbb{Q}$ ]. The ground-state properties such as deformation and superfluidity in neutron-rich $\mathrm{Zr}$ isotopes up to the drip line had been studied by employing the Skyrme-HFB method, and it had been predicted that $\mathrm{Zr}$ isotopes around $A=110$ are well deformed in the ground states [0]].

Spin-isospin responses of nuclei are described microscopically by the proton-neutron randomphase approximation (pnRPA) or the proton-neutron quasiparticle-RPA (pnQRPA) including the pairing correlations on top of the self-consistent Hartree-Fock (HF) or HF-Bogoliubov (HFB) mean fields employing the nuclear EDF. There have been many attempts to investigate the chargeexchange modes of excitation in stable and unstable nuclei [田]. These studies, however, are largely restricted to spherical systems, and the collective modes in deformed nuclei remain mostly unexplored. Thus, in the present contribution, I investigate the GT modes of excitation in the neutronrich $\mathrm{Zr}$ isotopes as examples of the deformed neutron-rich nuclei. And then, the deformation effect on the GT strength distribution and the $\beta$-decay half-lives are discussed.

\section{Theoretical model}

\subsection{Microscopic framework for spin-isospin responses of deformed neutron-rich nuclei}

To describe the nuclear deformation and the pairing correlations in the ground state, simultaneously, with a proper description of the weakly-bound nucleons, I solve the HFB equation in coordinate space [ए], [2]

$$
\left(\begin{array}{cc}
h^{q}(r \sigma)-\lambda^{q} & \tilde{h}^{q}(r \sigma) \\
\tilde{h}^{q}(r \sigma) & -\left(h^{q}(r \sigma)-\lambda^{q}\right)
\end{array}\right)\left(\begin{array}{c}
\varphi_{1, \alpha}^{q}(r \sigma) \\
\varphi_{2, \alpha}^{q}(r \sigma)
\end{array}\right)=E_{\alpha}\left(\begin{array}{c}
\varphi_{1, \alpha}^{q}(r \sigma) \\
\varphi_{2, \alpha}^{q}(r \sigma)
\end{array}\right)
$$

using cylindrical coordinates $r=(\rho, z, \phi)$. I assume axial and reflection symmetries to reduce the computational resources. Here, the superscript $q$ denotes $v$ (neutron, $t_{z}=1 / 2$ ) or $\pi$ (proton, 
$t_{z}=-1 / 2$ ). The mean-field Hamiltonian $h$ is derived from the Skyrme EDF. The pairing field $\tilde{h}$ is treated by using the density-dependent contact interaction,

$$
v_{\text {pair }}\left(r \sigma, r^{\prime} \sigma^{\prime}\right)=\frac{1-P_{\sigma}}{2} t_{0}^{\prime}\left[1+\frac{1}{2} \frac{\rho(r)}{\rho_{0}}\right] \delta\left(r-r^{\prime}\right)
$$

where $\rho(r)$ denotes the isoscalar density, $\rho_{0}=0.16 \mathrm{fm}^{-3}$, and $P_{\sigma}$ the spin exchange operator.

Since I consider the even-even mother (target) nuclei only, the time-reversal symmetry is assumed. A nucleon creation operator $\hat{\psi}_{q}^{\dagger}(r \sigma)$ at the position $r$ with the intrinsic spin $\sigma$ is then written in terms of the quasiparticle (qp) wave functions as

$$
\hat{\psi}_{q}^{\dagger}(r \sigma)=\sum_{\alpha}\left[\varphi_{1, \alpha}^{q}(r \bar{\sigma}) \hat{a}_{\alpha, q}^{\dagger}+\varphi_{2, \alpha}^{q *}(r \sigma) \hat{a}_{\alpha, q}\right] .
$$

The notation $\varphi(r \bar{\sigma})$ is defined by $\varphi(r \bar{\sigma})=-2 \sigma \varphi(r-\sigma)$.

Using the quasiparticle basis obtained as a self-consistent solution of the HFB equations ([2. (ل) the pnQRPA equation is solved

$$
\left[\hat{H}^{\prime}, \hat{O}_{i}^{\dagger}\right]|0\rangle=\omega_{i} \hat{O}_{i}^{\dagger}|0\rangle
$$

with $\hat{H}^{\prime}=\hat{H}-\lambda_{v} \hat{N}_{v}-\lambda_{\pi} \hat{N}_{\pi}$. The charge-changing QRPA phonon operators are defined as

$$
\hat{O}_{i}^{\dagger}=\sum_{\alpha \beta}\left[X_{\alpha \beta}^{i} \hat{a}_{\alpha, v}^{\dagger} \hat{a}_{\beta, \pi}^{\dagger}-Y_{\alpha \beta}^{i} \hat{a}_{\bar{\beta}, \pi} \hat{a}_{\bar{\alpha}, v}\right]
$$

where $\hat{a}_{\bar{\alpha}, q}$ is a quasiparticle annihilation operator of the time-reversed state of $\alpha$.

The $\mathrm{GT}^{ \pm}$transition strengths to the state $i$ with the $z$-component of angular momentum $K(K=0, \pm 1)$ are calculated as

$$
\begin{aligned}
B\left(\mathrm{GT}^{ \pm} ; i\right) & =\frac{g_{A}^{2}}{4 \pi}\left|\left\langle i\left|\hat{F}_{K}^{ \pm}\right| 0\right\rangle\right|^{2} \\
\left\langle i\left|\hat{F}_{K}^{ \pm}\right| 0\right\rangle & =\sum_{\alpha \beta}\left[X_{\alpha \beta}^{i}\left\langle\alpha \beta\left|\hat{F}_{K}^{ \pm}\right| \mathrm{HFB}\right\rangle-Y_{\alpha \beta}^{i}\left\langle\alpha \beta\left|\hat{F}_{K}^{\mp}\right| \mathrm{HFB}\right\rangle\right]
\end{aligned}
$$

under the quasi-boson approximation. The HFB vacuum is denoted as $|\mathrm{HFB}\rangle$, and $|\alpha \beta\rangle=\hat{a}_{\alpha, v}^{\dagger} \hat{a}_{\beta, \pi}^{\dagger}|\mathrm{HFB}\rangle$ is a 2qp excited state. The $\mathrm{GT}^{ \pm}$operators are given by

$$
\begin{aligned}
& \hat{F}_{K}^{+}=\sum_{\sigma \sigma^{\prime}} \int d r \hat{\psi}_{v}^{\dagger}\left(r \sigma^{\prime}\right)\left\langle\sigma^{\prime}\left|\sigma_{K}\right| \sigma\right\rangle \hat{\psi}_{\pi}(r \sigma), \\
& \hat{F}_{K}^{-}=\sum_{\sigma \sigma^{\prime}} \int d r \hat{\psi}_{\pi}^{\dagger}\left(r \sigma^{\prime}\right)\left\langle\sigma^{\prime}\left|\sigma_{K}\right| \sigma\right\rangle \hat{\psi}_{v}(r \sigma) .
\end{aligned}
$$

The transition-strength distributions can be presented as functions of the excitation energy $E_{T}$ with respect to ground state of the mother (target) nucleus

$$
R^{ \pm}\left(E_{T}\right)=\sum_{K} \sum_{i} \frac{\gamma / 2}{\pi} \frac{\left|\left\langle i\left|\hat{F}_{K}^{ \pm}\right| 0\right\rangle\right|^{2}}{\left[E_{T}-\left\{\omega_{i} \pm\left(\lambda_{v}-\lambda_{\pi}\right)\right\}\right]^{2}+\gamma^{2} / 4} .
$$




\subsection{Numerical details}

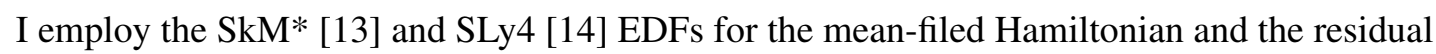
interaction in the $\mathrm{p}$-h channel. The pairing strength parameter $t_{0}^{\prime}$ is determined so as to approximately reproduce the experimental pairing gap of ${ }^{120} \mathrm{Sn}\left(\Delta_{\exp }=1.245 \mathrm{MeV}\right)$.

The pairing field is generated by using the density-dependent contact interaction of Eq. ([2.2). The strength parameter for the $T=0$ pairing interaction can be considered as a free parameter, because it dose not affect the ground-state properties, and it is active only at the dynamic level.

Because of the assumption of the axially symmetric potential, the $z$-component of the qp angular momentum, $\Omega$, is a good quantum number. Assuming time-reversal symmetry and reflection symmetry with respect to the $x-y$ plane, we have only to solve Eq. (D. $(\mathbb{D})$ for positive $\Omega$ and positive z. We use the lattice mesh size $\Delta \rho=\Delta z=0.6 \mathrm{fm}$ and a box boundary condition at $\rho_{\max }=14.7$ $\mathrm{fm}, z_{\max }=14.4 \mathrm{fm}$ to discretize the continuum states. The differential operators are represented by use of the 13-point formula of finite difference method. The quasiparticle energy cutoff is chosen at $E_{\mathrm{qp}, \mathrm{cut}}=60 \mathrm{MeV}$ and the quasiparticle states up to $\Omega^{\pi}=31 / 2^{ \pm}$are included.

We introduce an additional truncation for the pnQRPA calculation, in terms of the 2qp energy as $E_{\alpha}+E_{\beta} \leq 60 \mathrm{MeV}$. This reduces the number of 2qp states to, for instance, about 30000 for the $K^{\pi}=0^{+}$excitation of the $\mathrm{Zr}$ isotopes. The number of 2qp states included in the calculation is large enough to satisfy the Ikeda sum-rule values to an accuracy of $1 \%$. The calculation of the QRPA matrix elements in the qp basis, and diagonalization of the QRPA matrix are performed in the parallel computers as in Ref. [15]].

\section{Results and discussion}

First, I am going to discuss the roles of the neutron excess on the GTGR. One could expect the enhancement in the $\mathrm{GT}^{-}$transition strengths according to the Ikeda sum rule:

$$
S_{-}-S_{+}=3(N-Z)
$$

where $S_{ \pm}$is a sum of the $\mathrm{GT}^{ \pm}$strengths, and the $\mathrm{GT}^{+}$strengths are strongly suppressed due to the Pauli effect. Furthermore, because of the imbalanced Fermi levels of neutrons and protons, the number of " $0 \hbar \omega$ " 2qp excitation increases. One can thus expect a strong collectivity for the $\mathrm{GT}^{-}$modes of excitation. Figure प(a) shows the development of the collectivity of the GTGR associated with the neutron excess. Plotted here is the mean energy difference of the $\mathrm{GT}^{-}$strength distribution due to the residual interaction; $\Delta E=\bar{E}_{\mathrm{QRPA}}-\bar{E}_{\mathrm{HFB}}$. The excitation energy $\bar{E}$ is defined by the moment:

$$
\bar{E}=\frac{\int d E^{\prime} E^{\prime} R^{-}\left(E^{\prime}\right)}{\int d E^{\prime} R^{-}\left(E^{\prime}\right)}
$$

Both the SkM* and SLy4 interactions give a repulsive contribution to generation of the GTGR. Note that the Laudau-Migdal parameter $g_{0}^{\prime}$ is 0.93 and 0.90 for the SkM* and SLy4 EDF, respectively. As increasing the neutron excess, the GTGR is shifted more up in energy.

To see what is happening in the extreme case, I show in Fig. W(b) the $\mathrm{GT}^{-}$strength distributions in ${ }^{140} \mathrm{Zr}$. The nucleus ${ }^{140} \mathrm{Zr}$ is considered to be located close to the drip line; the Fermi level of neutrons is $-0.10 \mathrm{MeV}$ with $\mathrm{SkM}^{*}$. Without the residual interaction, indicated by the dashed line 

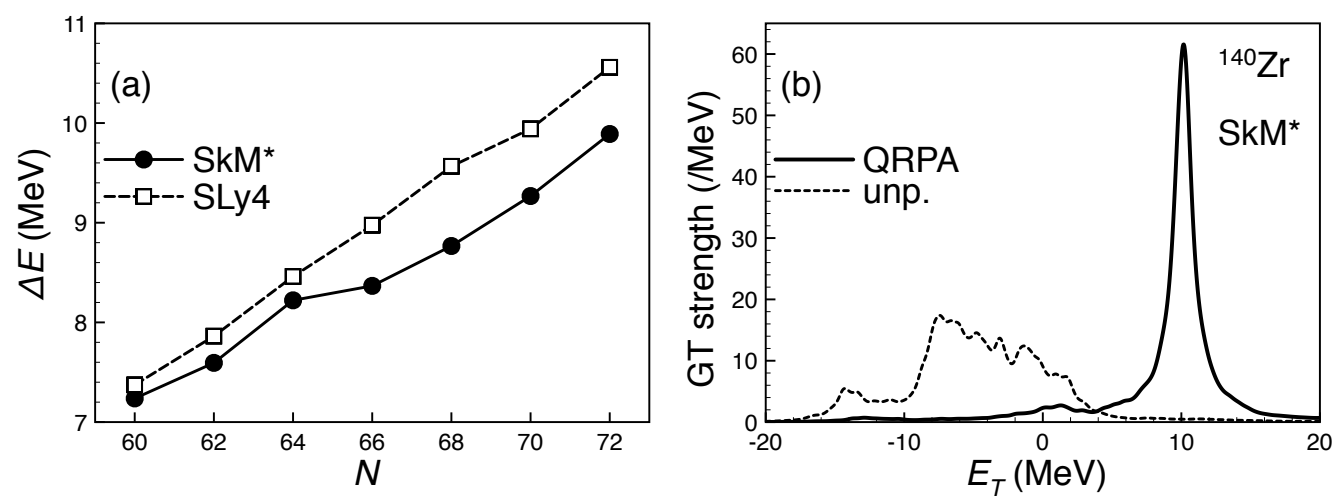

Figure 1: (a) Shift of the excitation energy of GTGR due to the residual interaction. See text for details. (b) GT $^{-}$strength (in unit of $g_{A}^{2} / 4 \pi$ ) distributions of the drip-line nucleus ${ }^{140} \mathrm{Zr}$ with and without the residual interaction as functions of the excitation energy with respect to the ground state of the target nucleus.
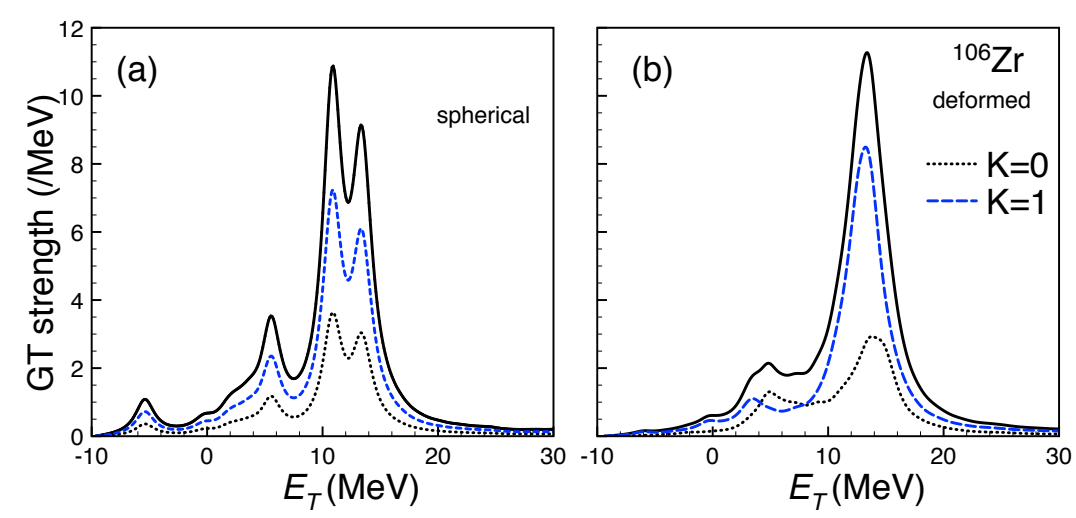

Figure 2: $\mathrm{GT}^{-}$strength (in unit of $g_{A}^{2} / 4 \pi$ ) distributions of the ${ }^{106} \mathrm{Zr}$ as functions of the excitation energy $E_{T}$. The SLy4 EDF is employed without the $T=0$ pairing interaction. The strengths are smeared with $\gamma=2$ $\mathrm{MeV}$. The $K=0$ and $K=1$ strengths are denoted by the dotted and dashed lines. For the $K=1$ strength, the $K= \pm 1$ components are summed up. (a) Strength distribution obtained assuming the nucleus spherical. (b) Strength distribution for the prolately-deformed ground state.

in the figure, the strength distributions are fragmented in an energy region $-10-0 \mathrm{MeV}$, and a bump structure around $-15 \mathrm{MeV}$ is also seen. This means that many 2qp excitations are available for constructing the $\mathrm{GT}^{-}$modes of excitation. When turing on the residual interaction, one sees a prominent peak at $10 \mathrm{MeV}$. The GTGR in this nucleus collects almost all the strengths in a narrow resonance. The mean energy difference associated with the residual interaction reaches 14.0 MeV, indicating a quite strong collectivity. In the light drip-nuclei such as ${ }^{8} \mathrm{He}$, the GTGR appears below the ground state of the mother nucleus [[D]]. The strong collectivity in the heavy systems, however, prevents the occurrence of the super-allowed GTGR even with a high asymmetry $\alpha=(N-Z) / A=0.43$.

Next, I am going to discuss the deformation effects. Figure $\square$ shows the $\mathrm{GT}^{-}$strength distributions in ${ }^{106} \mathrm{Zr}$ calculated employing the SLy4 EDF. The ground state is prolately deformed 


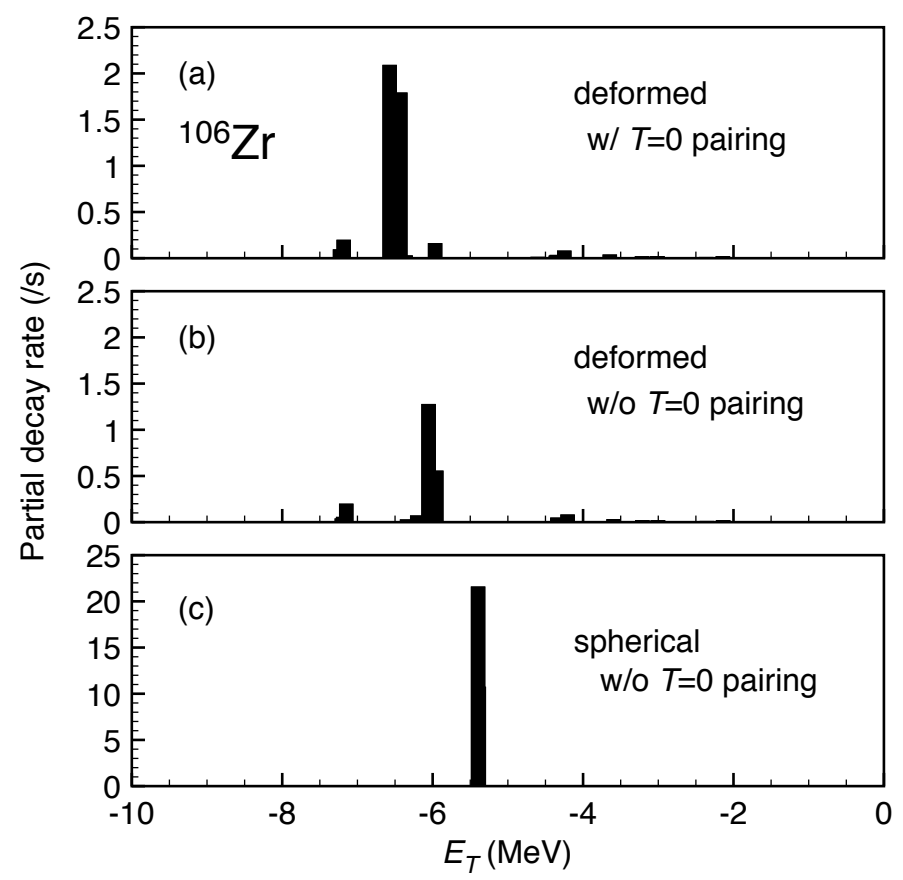

Figure 3: Partial decay rates in ${ }^{106} \mathrm{Zr}$ with the SLy4 EDF. (a) Calculated with the $T=0$ pairing interaction on the prolately-deformed ground state. (b) Calculated without the $T=0$ pairing interaction on the prolatelydeformed ground state. (c) Calculated without the $T=0$ pairing interaction assuming the nucleus spherical.

in the calculation; $\beta_{2}=0.39$. To see the roles of deformation, I show in Fig. $\mathbb{Z}(\mathrm{a})$ the strength distribution obtained assuming the nucleus spherical. Namely, the HFB equation (D. with a constraint for the deformation $\beta_{2}=0$. In both cases, one sees the GTGR around 12-16 $\mathrm{MeV}$ together with a bump around $5 \mathrm{MeV}$. Since the GT operator (2.8) carries the total angular momentum $J=1$, one has the $K=0$ and \pm 1 components. For the spherical nuclei possessing the rotational symmetry, the angular momentum is a good quantum number. So, the strengths of $K=0$ and $K= \pm 1$ components are the same, as seen in Fig. $\mathbf{Z}$ (a). For the deformed nuclei, such as ${ }^{106} \mathrm{Zr}$, the angular momentum is no more a good quantum number. Thus, one sees a $K$-splitting for the giant dipole resonance, for instance [3]. One can also see a $K$-splitting for the GT excitation as shown in Fig. D(b) though its splitting is tiny, in particular, for the GTGR peak.

In Fig. \, one can see a distinct deformation effect: The low-lying strengths concentrated around $-6 \mathrm{MeV}$ in Fig. $\mathbb{Z}(\mathrm{a})$ get spread over in Fig. $\mathbb{Z}(\mathrm{b})$. Since the $\beta$-decay rate is predominantly governed by the low-lying GT states, it strongly affects the $\beta$-decay properties. In what follows, I am going to discuss the deformation effect on the $\beta$-decay half-lives.

For the neutron-rich $\mathrm{Zr}$ isotopes around $N=66-70$, the $\beta$-decay half-lives were measured experimentally [ [Q Q Q $]$ ]. Figure B] shows the partial decay rates of ${ }^{106} \mathrm{Zr}$ obtained by employing the SLy4 EDF. For the $T=0$ pairing interaction, the density-independent contact force is employed for simplicity. The strength of the paring interaction was determined so as to reproduce the measured $\beta$-decay half-life of ${ }^{100} \mathrm{Zr}\left(T_{1 / 2}=7.1 \mathrm{~s}\right)$. For the axial-vector coupling constant, I used the effective one; $\left(g_{\mathrm{A}}\right)_{\mathrm{eff}}=1.0$. 
For the spherical configuration, one sees a state at $E_{T}=-5.5 \mathrm{MeV}$ in Fig. B](c), which appears as a low-lying "resonance" in Fig. $\mathbb{D}($ a) due to the artificial smearing width. This prominent state leads to a short half-life of $0.031 \mathrm{~s}$. When considering the deformation, the state at $-5.5 \mathrm{MeV}$ gets fragmented as shown in Fig. B](b). The deformation-induced fragmentation reduces the decay rates and the calculated half-life is $0.41 \mathrm{~s}$. The low-lying GT states are sensitive to the detail of the shell structure and the residual interactions. Figure B(a) shows the partial decay rates calculated taking into account the $T=0$ pairing. One sees that the low-lying states are pushed down in energy due to the attractive $T=0$ pairing interaction, which enhances the $\beta$-decay rate; the calculated $\beta$-decay half-life is $0.22 \mathrm{~s}$. Note that the observed $\beta$-decay half-life is $0.186_{-0.010}^{+0.011} \mathrm{~s}$. The prominent state at $E_{T} \simeq-6 \mathrm{MeV}$ in Fig. $\mathbb{Z}(\mathrm{b})$ is mainly constructed by a $v[413] 5 / 2 \otimes \pi[413] 7 / 2$ excitation satisfying the selection rule:

$$
\left|\left\langle\pi\left[N n_{3} \Lambda\right] \Omega=\Lambda \pm 1 / 2\left|t_{-} \sigma_{ \pm 1}\right| v\left[N n_{3} \Lambda\right] \Omega=\Lambda \mp 1 / 2\right\rangle\right|=\sqrt{2} .
$$

The occupation probability of a $v[413] 5 / 2$ orbital is 0.31 . Thus, this 2qp excitation is a p-p type excitation, and is then strongly affected by the $T=0$ pairing interaction.

\section{Summary}

I discussed the low-lying GT modes of excitation and GTGR in the neutron-rich Zr isotopes, putting an emphasis on the roles played by the nuclear deformation. The deformation-induced fragmentation of the strength distribution in the low excitation-energy region has a strong impact on the $\beta$-decay properties, while the $K$-splitting is small for the GTGR. The attractive $T=0$ pairing interaction lowers the frequency of the low-lying GT mode and enhances the GT transition strength when the 2qp excitation generating the low-lying mode satisfies the selection rule and is a p-p type excitation. Therefore, the effect of $T=0$ pairing is very sensitive to the shell structure around the Fermi level. To reproduce the experimental data of $\beta$-decay half-lives, the fragmentation of the GT strength distribution associated with the nuclear deformation is important together with the $T=0$ pairing interaction. The nuclear EDF-based QRPA works better for the deformed systems than for the spherical systems, where one needs to go beyond the RPA to obtain the fragmentation of the strengths.

\section{Acknowledgements}

This work was supported by the JSPS KAKENHI (Grants No. 25287065, and No. 16K17687). The numerical calculations were performed on SR16000 and CRAY XC40 at the Yukawa Institute for Theoretical Physics, Kyoto University, and on COMA (PACS-IX) at the Center for Computational Sciences, University of Tsukuba.

\section{References}

[1] F. Osterfeld, Rev. Mod. Phys. 64, 491 (1992).

[2] F. T. Baker et al., Phys. Rep. 289, 235 (1997).

[3] B. L. Berman, and S. C. Fultz, Rev. Mod. Phys. 47, 713 (1975). 
[4] N. Paar, D. Vretenar, E. Khan, and G. Colò, Rept, Prog. Phys. 70, 691 (2007).

[5] K. Langanke and G. Martínez-Pinedo, Rev. Mod. Phys. 75, 819 (2003).

[6] M. V. Stoitsov, J. Dobaczewski, W. Nazarewicz, S. Pittel, and D. J. Dean, Phys. Rev. C 68, 054312 (2003).

[7] J. Erler, N. Birge, M. Kortelainen, W. Nazarewicz, E. Olsen, A. M. Perhac, and M. Stoitsov, Nature 486, 509 (2012).

[8] S. Nishimura et al., Phys. Rev. Lett. 106, 052502 (2011).

[9] G. Lorusso et al., Phys. Rev. Lett. 114, 192501 (2015).

[10] A. Blazkiewicz, V. E. Oberacker, A. S. Umar, and M. Stoitsov, Phys. Rev. C 71, 054321 (2005).

[11] A. Bulgac, Preprint No. FT-194-1980, Institute of Atomic Physics, Bucharest (1980), arXiv:nucl-th/9907088.

[12] J. Dobaczewski, H. Flocard, and J. Treiner, Nucl. Phys. A422 (1984) 103.

[13] J. Bartel, P. Quentin, M. Brack, C. Guet, and H.-B. Håkansson, Nucl. Phys. A386, 79 (1982).

[14] E. Chabanat, P. Bonche, P. Haensel, J. Meyer, and R. Schaeffer, Nucl. Phys. A635, 231 (1998).

[15] K. Yoshida and T. Nakatsukasa, Phys. Rev. C 88, 034309 (2013).

[16] K. Yoshida, Prog. Theor. Exp. Phys. 2013 113D02 (2013).

[17] H. Sagawa, I. Hamamoto, and M. Ishihara, Phys. Lett. B303 215 (1993). 